\title{
Brain dynamics in the comprehension of action-related language. A time-frequency analysis of mu rhythms
}

\author{
Iván Moreno a,*, Manuel de Vega a , Inmaculada León ${ }^{\text {a }}$, Marcel Bastiaansen ${ }^{\text {b }}$, \\ Ashley Glen Lewis ${ }^{\mathrm{c}, \mathrm{d}}$, Lilla Magyari ${ }^{\mathrm{e}}$ \\ a Department of Cognitive Psychology, University of La Laguna, Tenerife, Spain \\ ${ }^{\mathrm{b}}$ Academy for Leisure, NHTV Breda University of Applied Sciences, The Netherlands \\ c Neurobiology of Language Department, Max Planck Institute for Psycholinguistics, Nijmegen, The Netherlands \\ ${ }^{\mathrm{d}}$ Radboud University, Donders Institute for Brain, Cognition and Behaviour, Centre for Cognitive Neuroimaging, Nijmegen, The Netherlands \\ e Language E' Cognition Department, Max Planck Institute for Psycholinguistics, Nijmegen, The Netherlands
}

\section{A R T I C L E I N F O}

\section{Article history:}

Accepted 2 January 2015

Available online 9 January 2015

Keywords:

Mu rhythms

Beta rhythms

Embodied meaning

Action language

Perceptive language

\begin{abstract}
A B S T R A C T
EEG mu rhythms $(8-13 \mathrm{~Hz})$ recorded at fronto-central electrodes are generally considered as markers of motor cortical activity in humans, because they are modulated when participants perform an action, when they observe another's action or even when they imagine performing an action. In this study, we analyzed the time-frequency (TF) modulation of mu rhythms while participants read action language ("You will cut the strawberry cake"), abstract language ("You will doubt the patient's argument"), and perceptive language ("You will notice the bright day"). The results indicated that mu suppression at fronto-central sites is associated with action language rather than with abstract or perceptive language. Also, the largest difference between conditions occurred quite late in the sentence, while reading the first noun, (contrast Action vs. Abstract), or the second noun following the action verb (contrast Action vs. Perceptive). This suggests that motor activation is associated with the integration of words across the sentence beyond the lexical processing of the action verb. Source reconstruction localized mu suppression associated with action sentences in premotor cortex (BA 6). The present study suggests (1) that the understanding of action language activates motor networks in the human brain, and (2) that this activation occurs online based on semantic integration across multiple words in the sentence.
\end{abstract}

(c) 2015 Elsevier Inc. All rights reserved.

\section{Introduction}

Action-related language is currently an active field of research within the so-called embodied semantic approach to linguistic meaning. This approach claims that meaning is grounded in our perceptive and motor interactions with the world. From a neurobiological point of view, embodied theories postulate that language comprehension partially relies on modality-specific neural systems for perception and action (Barsalou, 1999; Barsalou et al., 2003; Barsalou, 2008; Damasio et al., 2004; Fischer and Zwaan, 2008; Glenberg and Gallese, 2011; de Vega et al., 2008). Neuroimaging studies have reported activations in the motor and premotor brain regions during the comprehension of action-related language. Some of these studies found motor activation when people process action verbs presented in isolation (Hauk and Pulvermüller, 2004; Pulvermüller, 1996; Pulvermüller et al., 2005; Rizzolatti and Luppino, 2001), and others have found similar motor activation when people process action-related sentences (Aziz-Zadeh

\footnotetext{
* Corresponding author at: Department of Cognitive Psychology, University of La Laguna, Campus de Guajara, s/n, La Laguna, Tenerife 38205, Spain.

E-mail address: ivanzevenzui@hotmail.com (I. Moreno).
}

et al., 2006; Moody and Gennari, 2010; Raposo et al., 2009; Tettamanti et al., 2005; Urrutia et al., 2012; De Vega et al., 2014).

Such results have been taken as evidence that motor networks in the brain are involved in the processing of action-related language, although some important issues still remain unsolved (de Vega et al., 2008; Mahon and Caramazza, 2008). One of these issues is the need to determine whether language-induced motor activations are exclusively lexical or are dependent to some extent on the semantic context. One possibility is that the activation of the motor cortex occurs automatically about 150-200 ms after the onset of action verbs, as part of their lexical processing (Pulvermüller et al., 2005; 2009; Boulenger et al., 2006; Buccino et al., 2001; Nazir et al., 2008; Sato et al., 2008). Another alternative is that the activation of the motor cortex associated with reading is a non-automatic, context-dependent process, involving lexical-semantic integration across the sentence. The latter proposal is based on neuroimaging (Moody and Gennari, 2010; Schuil et al., 2013; Raposo et al., 2009; Urrutia et al., 2012), and brain stimulation studies (Cacciari et al., 2011; Buccino et al., 2005; Tomasino et al., 2008; Papeo et al., 2009). For example, in a fMRI experiment Raposo et al. (2009) reported that action verbs activate motor regions when they appear in concrete literal sentences (e.g., kick the ball), whereas the 
same verbs in idiomatic sentences do not activate motor regions (e.g., kick the bucket). In the same vein, Cacciari et al. (2011) employing single-pulse TMS on the left leg-related motor cortex while participants read leg-related action verb embedded in sentences, reported modulations of cortico-spinal excitability only when the action verbs appeared in a literal or metaphorical context, but not when the same verbs appeared in idioms.

Given the poor temporal resolution of hemodynamic neuroimaging and single-pulse TMS techniques, the above studies of language comprehension mainly provide a neuroanatomical view on motor activation in the brain. The present study uses a different strategy by recording moment-to-moment changes in mu and beta oscillatory rhythms in the EEG, which are generally considered as markers of motor processes in the brain (Pfurtscheller, 1992; Pfurtscheller et al., 1997; Pfurtscheller and Lopes da Silva, 1999). Mu rhythms are $8-13 \mathrm{~Hz}$ oscillations generated in the motor and premotor cortex, which provide a high-resolution window onto the temporal dynamics of motor activation (Gastaut, 1952; Muthukumaraswamy et al., 2004). Specifically, mu synchronization reflects neural activity correlating with a deactivated motor cortex, whereas mu suppression or desynchronization can be attributed to an increase in neural activity of the motor and premotor cortex (Kuhlman, 1978; Pfurtscheller et al., 1997). Thus, these rhythms become suppressed or desynchronized when participants move, observe other's movements, or imagine their own movements, especially if these movements are manual (Cochin et al., 1998; McFarland et al., 2000a, 2000b; Pfurtscheller et al., 2006; Pineda, 2005).

Recently, some studies have reported that mu rhythms, and to some extent beta rhythms, can also be modulated by action-related language. Thus, Moreno et al. (2013) asked their participants to listen to sentences referring to manual actions (e.g., Now, I cut the bread) or abstract sentences (e.g., Now, I doubt the plan), and also to watch video clips of an actress performing simple manual actions. The results indicated that both action language and action videos caused $\mathrm{mu}(8-13 \mathrm{~Hz})$ and low beta $(15-20 \mathrm{~Hz})$ power suppression at fronto-central electrodes, whereas abstract language did not, confirming that understanding action language activates motor networks in the brain. However, Moreno et al.'s study did not allow for assessing the temporal evolution of the mu and beta rhythms. In another study, van Elk et al. (2010) used time-frequency analysis while participants read action sentences and observed larger suppression in mu and beta rhythms in response to the action verb ("swimming") paired with an animal agent (e.g., duck) compared to a human agent (e.g., the woman), suggesting that motor resonance is a context-dependent and integrative phenomenon, as it depends on the previously mentioned agent. One drawback of the study, however, is that the absence of non-action sentences as a contrasting condition does not allow the conclusion that these timeresolved mu and beta rhythms are exclusively modulated by action language. A different research strategy was employed by Fargier et al. (2012). They trained participants to associate new words with action related videos or with visual motion videos in two sessions. After the second training session they found mu suppression for actionassociated words presented alone at fronto-central sites.

The present study goes one step further than the previous brain dynamics studies on action language. Our first goal was to determine whether motor processes in the brain are specific to action language. We therefore examined the modulation of mu and beta rhythms by contrasting action sentences with non-action sentences. Specifically, we employed three types of contents: action sentences referring to concrete manual actions, abstract sentences referring to mental states or processes, and perceptive sentences referring to sensory events. All sentences were written in the second person because the pronoun "you" most likely induces an egocentric perspective (Brunyé et al., 2009), and in the future tense because preparing to perform an action elicits stronger motor activity than an action already performed (Candidi et al., 2010). We expected to find mu and beta modulation (suppression) in action sentences but not in abstract sentences, confirming our previous findings (Moreno et al., 2013). We were however particularly interested in the contrast between action sentences and perceptive sentences, which to our knowledge has never been used in conjunction with an analysis of EEG oscillations. One possibility is that only action sentences modulate mu rhythms, which would indicate that motor cortical activity is very specific to the semantics of action sentences. A second possibility is that perceptive sentences also modulate mu rhythms to some extent, perhaps because the sensory processes described by the sentences also involve motor actions like head movement or body postural changes, or because perception and action are closely related processes in the brain. If this is the case, then we should observe equally strong mu and/or beta suppression for the action and the perception conditions over sensorimotor electrodes. Finally, given that perceptive sentences describe mostly visual events, we may also expect differential activation of cortical areas responsible for visual processing (i.e. occipital and inferior-temporal lobes), thereby producing differential alpha suppression over these areas (Bastiaansen and Brunia, 2001; Pulvermüller et al., 1999; Klimesch et al., 2007; Weisz et al., 2011; Woertz et al., 2004).

Our second goal was to examine whether the activity in the motor cortex is exclusively triggered by the action verbs, involving purely lexical and automatic motor resonance, or whether motor cortical activity requires the integration of linguistic units (verb + noun in our materials) to build up a specific motor simulation of the complete action. With this aim in mind we employed time-frequency analysis (hereafter TF) of mu and beta rhythms across the whole sentence. The action sentences in this study were written in such a way that the action was not entirely specified at the verb, but was completed by the two nouns that immediately followed the verb (see Table 1 ). If mu/beta suppression occurs exclusively at the action verb this would support the idea that modulation of motor activity is purely lexical. By contrast if $\mathrm{mu}$ /beta suppression occurs in the nouns following the verb, we can assume that if a context is supplied, modulations of motor activity result from integration of meaning across the sentence.

We also presented participants incongruent action sentences to have additional information about the integration of meaning across sentences. Incongruent action sentences used the same verbs as congruent action sentences, but they included an object name that made them non-sensical. Processes of semantic integration have typically been associated with the N400 ERP component, a negative deflection found over centro-parietal electrodes, peaking approximately $400 \mathrm{~ms}$ after word onset (Kutas and Hillyard, 1984). We therefore could expect an N400 effect after the onset of the noun directly following the verb where the sentence congruence or incongruence emerges.

Table 1

Example stimulus materials. The verb, the Noun1 and the Noun2 are marked in bold.

\begin{tabular}{ll}
\hline Congruent Action sentences & \\
Spanish & Abrirás la puerta de la habitación \\
English & You will open the door of the room
\end{tabular}

English

You will open the door of the room

Congruent Perceptive sentences Spanish

English

Escucharás la orden del maestro You will hear the teacher's command

Congruent Abstract sentences Spanish

English

Dudarás del diálogo del paciente You will doubt the patient's dialog

Incongruent Action sentences Spanish

English

Abrirás el color del techo You will open the color of the roof

Incongruent Perceptive sentences Spanish

Escucharás el retrato del álbum English You will hear the photo of the book 
If semantic integration of motor information occurs across the sentence, we can also expect a congruence effect on the oscillatory rhythms (modulation of mu or beta) when processing this noun. By contrast, if the verb is the critical linguistic unit associated with the engagement of motor processes then there should be no difference between congruent and incongruent sentences in the $\mathrm{mu}$ /beta rhythms.

Our final goal was to estimate the neural sources of the significant $\mathrm{mu} /$ beta effects. Most studies analyzing $\mathrm{mu}$ /beta rhythms assume that their fronto-central distribution at the scalp implies that they are generated in the (pre)motor cortex. However, we want to be more specific by employing source estimation algorithms to estimate the actual neuronal generators of the effects. Eventually we expect to be able to reveal differential brain sources for action sentences (motor networks) and for perceptive sentences (visual networks).

\section{Methods}

\section{Participants}

Twenty-eight undergraduate students of Psychology at the University of La Laguna participated in this study. Data from 4 participants were discarded due to excessive eye-blinks or muscle artifacts, leaving 24 participants for analysis (9 males, 15 females; mean age $=24.5$ years). All participants were right-handed according to the lateralization coefficient of the Edinburgh Handedness Inventory (Mean = 77.16; SD = 16.67), had Spanish as their mother tongue, and reported no neurological diseases, visual problems or intake of medication. All participants received course credit for volunteering.

\section{Materials and design}

A 2 Congruence (congruent vs. incongruent) $\times 3$ Sentence Type (action vs perceptive vs abstract) within-participants experimental design was used. A total of 225 sentences were created, of which 120 were congruent and 80 were incongruent. The congruent sentences consisted of: 40 congruent action sentences (CA) referring to concrete manual actions, 40 congruent perceptive sentences (CP) referring to sensory processes, and 40 congruent abstract sentences (ABS) referring to mental states or processes. The incongruent sentences consisted of: 40 incongruent action sentences (IA) referring to impossible hand actions and 40 incongruent perceptive sentences (IP) referring to out of the ordinary perceptive experiences (see Table 1). We did not include incongruent abstract sentences because they were not necessary for our experimental contrasts. The remaining 25 sentences were catch trials, similar to the experimental trials but followed by a coherence judgment prompt. All sentences had the following sequence: the verb in the second person and future tense; a function word; the first noun; a function word, and the second noun, as illustrated in Table 1.

Both, the verbs and the nouns were matched on several lexical factors across the experimental conditions: number of syllables (2 or 3 ), full-word frequency, age of acquisition, and familiarity. The word frequency was obtained in the Spanish word frequency dictionary (Davis and Perea, 2005), and the age of acquisition and familiarity scores were obtained in two norming studies performed with 38 and 32 participants, respectively. In each norming study, participants received a booklet with 400 nouns ( 200 sentence first-nouns, and 200 sentence second-nouns), and 24 verbs ( 8 action verbs, 8 perceptive verbs, and 8 abstract verbs) in random order. In the first study, participants were asked to estimate the age at which they learnt the item, using a 7-point Likert scale: from 1 (earlier than 2 years old) to 7 (later than 12 years old). In the second study, participants were instructed to rate how familiar the items were on a 7-point Likert scale: from 1 (very uncommon) to 7 (very common). Given that Levene's test for inequality of variances of the scores was not significant, t-tests assuming equal variances were applied. Pairwise comparisons confirmed that verbs and nouns did not differ significantly in any of the critical lexical factors among the experimental conditions (see Table 2). A total of 8 verbs were used per condition (action, perceptive and abstract) each appearing in five congruent sentences, and in five incongruent sentences.

Given that we were interested in manipulating congruence, namely how plausible or feasible the action and the perceptive sentences were, we carried out another norming study with 25 new participants. They received a booklet with 160 sentences (40 CA, 40 IA, 40 CP and 40 IP) in random order. Participants were asked to rate using a 7-point Likert scale how feasible the events described by the sentences were: from 1 (impossible) to 7 (perfectly possible). As expected CA sentences were judged more feasible than IA ( $M=4.8, S D=0.54$ vs. $M=1.31$, $\mathrm{SD}=0.86$ respectively; $t(39)=20.01, p<.001)$, and CP were judged more feasible than IP $(\mathrm{M}=4.75, \mathrm{SD}=0.33$ vs. $\mathrm{M}=1.21, \mathrm{SD}=0.35$ respectively; $t(39)=45.63, p<.001)$.

\section{Procedure}

The experiment was controlled by E-PRIME (version 2.0.10.242, Psychology Software Tools; Schneider et al., 2002). During the experiment, participants were seated in a sound and electrically shielded room at an approximate distance of between 75 and $85 \mathrm{~cm}$ from the screen. The sentences were presented in random order. Each sentence was presented word by word in the center of the screen and subtended a visual angle of $3.4^{\circ}$ vertically. Words were presented in black characters (Arial font size 26 points) on a gray background, according to the temporal sequence shown in Fig. 1. To keep participants' attention on the task, they were prompted to perform coherence judgments (is it coherent? Yes/No) in 25 catch trials, appearing at random during the EEG session. Participants responded using the keyboard by pressing the "L" key with their right index finger or the " $\mathrm{A}$ " key with their left index finger, for Yes and No responses respectively. Each sentence remained on the screen until the participant responded or for a maximum of $3000 \mathrm{~ms}$. After the participants responded, the screen went blank for $4000 \mathrm{~ms}$ and then an asterisk indicated that the next trial would start in 2000-2500 ms.

Participants were instructed to sit as still as possible in a comfortable chair with instructions to remain relaxed and to minimize blinking during the experiment when the words were presented on the screen. They were asked to carefully read all the sentences that were presented because sometimes they had to indicate whether the sentence was coherent or not. The experiment started with 8 practice trials, and was followed by a single block of 225 trials, taking about $36 \mathrm{~min}$.

Table 2

Verbs and nouns lexical features: means and standard deviations.

\begin{tabular}{lllll}
\hline & $\begin{array}{l}\text { Lexical } \\
\text { frequency }\end{array}$ & $\begin{array}{l}\text { Word } \\
\text { length }\end{array}$ & $\begin{array}{l}\text { Age of } \\
\text { acquisition }\end{array}$ & $\begin{array}{l}\text { Subjective } \\
\text { familiarity }\end{array}$ \\
\hline Verbs & & & & \\
$\quad$ Action verbs & $26.34(29.39)$ & $2.12(0.35)$ & $2.63(0.45)$ & $5.91(0.62)$ \\
Perceptive verbs & $23.37(22.55)$ & $2.62(0.52)$ & $3.71(1.33)$ & $4.69(2.16)$ \\
Abstract verbs & $24.24(27.04)$ & $2.87(0.35)$ & $3.75(0.83)$ & $5.86(0.55)$ \\
& & & & \\
First noun & & & & \\
Congruent Action & $42.21(64.02)$ & $2.04(0.54)$ & $3.00(0.96)$ & $5.81(1.34)$ \\
Congruent Perceptive & $38.07(34.62)$ & $2.45(0.50)$ & $3.30(1.40)$ & $5.65(1.28)$ \\
Congruent Abstract & $44.11(41.87)$ & $2.65(0.53)$ & $4.34(1.25)$ & $5.45(0.86)$ \\
Incongruent Action & $41.96(44.45)$ & $2.50(0.51)$ & $3.20(1.19)$ & $5.94(0.78)$ \\
Incongruent Perceptive & $41.43(53.64)$ & $2.40(0.54)$ & $3.47(1.11)$ & $5.55(0.94)$ \\
Second noun & & & & \\
Congruent Action & $55.76(88.14)$ & $2.45(0.55)$ & $2.86(1.25)$ & $5.70(1.41)$ \\
Congruent Perceptive & $48.65(112.38)$ & $2.45(0.60)$ & $3.14(1.02)$ & $5.40(1.77)$ \\
Congruent Abstract & $105.87(117.95)$ & $2.70(0.46)$ & $3.15(1.40)$ & $5.72(0.89)$ \\
Incongruent Action & $42.22(62.97)$ & $2.52(0.64)$ & $3.21(1.13)$ & $5.54(0.96)$ \\
Incongruent Perceptive & $45.46(116.76)$ & $2.45(0.60)$ & $2.98(1.25)$ & $5.46(1.49)$ \\
\hline
\end{tabular}




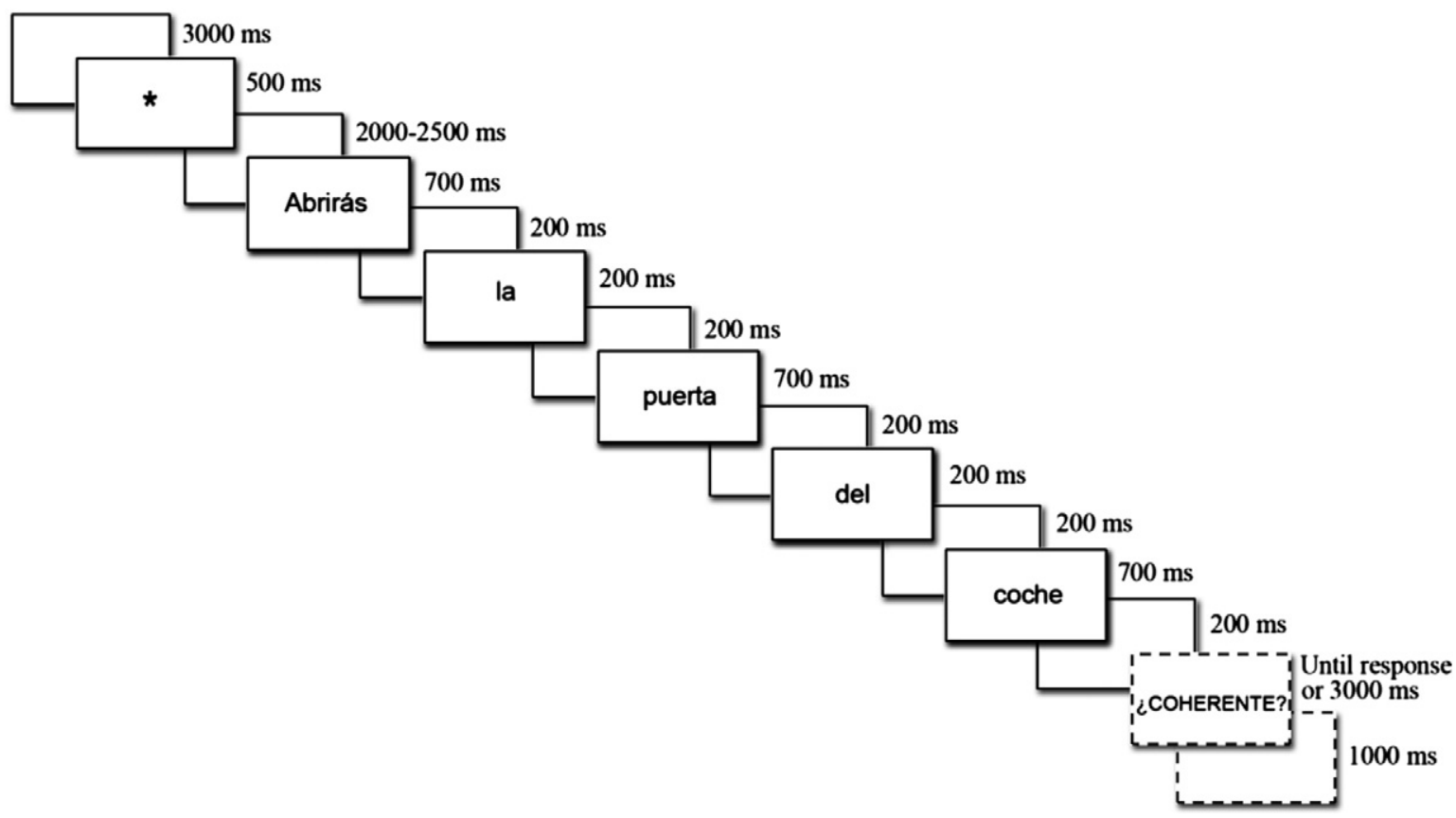

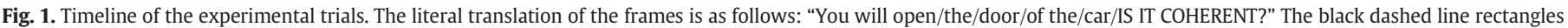
represent the additional events only occurring in the catch trials.

\section{Recording}

Participants' EEG was recorded continuously by a Compumedics Neuroscan (version 4.5) system from 64 active electrodes mounted in a Quick-Cap elastic cap, following the 10/20 system. Right mastoid was used as implicit reference, and AFz served as ground. The signal was amplified (SynAmps2) and digitized at a sampling rate of $1000 \mathrm{~Hz}$. A band-pass filter from 0.1 to $30 \mathrm{~Hz}$ and a $50 \mathrm{~Hz}$ notch filter were applied online. Re-referencing to the average of the two mastoids (right and left mastoids) was performed offline. The impedance of the electrodes was kept below $10 \mathrm{k} \Omega$. Blinks and eye movements were monitored using bipolar horizontal and vertical EOG derivations via two pairs of electrodes, one pair attached to the external canthi, and the other one to the infra orbital and supra orbital regions of the right eye.

\section{Data analysis}

The catch trials were submitted to 2 Congruence (Incongruent, Congruent) $\times 2$ Sentence Type (Action, Perceptive) ANOVAs for percentage of "yes" responses. These trials, however, were not included in the EEG analysis. The epochs used for the ERP analysis and the timefrequency analysis ( -2000 to $4000 \mathrm{~ms}$ relative to the onset of the verb) were checked for blinks and eye movements, identified by abrupt changes in the EOG waves. These epochs were submitted to an artifact reduction algorithm, which subtracted the eye movements from the ongoing EEG, improving it before averaging. Data from four participants were excluded from the analysis due to the excessive number of blinks and motor artifacts (facial muscle activity) identified during the session. After the automatic correction of blinks, the remaining data were carefully inspected and trials which still showed any kind of artifacts were rejected. The average number of accepted trials did not significantly differ among conditions $(F(1,23)=1.16, p>.05)$. The mean and standard deviations of number of accepted trials were: $\mathrm{M}=70.42 \%$ ( $\mathrm{SD}=$ 5.89) for the CA, $M=72.5 \%$ ( $S D=5.83$ ) for the $A B S, M=73.02 \%$ $(S D=5.69)$ for the $C P, M=69.27 \%(S D=6.37)$ for the $I A$, and $M=$ $70.94 \%(\mathrm{SD}=5.83)$ for IP.
Event-related potential (ERP) analysis

The main purpose of the ERP analysis was to explore the congruence effects, both in action sentences (contrast IA vs CA) and perceptive sentences (contrast IP vs. CP), so ABS sentences were not included in the ERP analysis. The congruence manipulation was such that the critical word was always the first noun (hereafter Noun1), immediately following the main verb; for instance, "door" in the sentence "you will open the door of the room" (see Table 1). The data were segmented individually for the CA, IA, CP, and IP conditions from -200 to $1000 \mathrm{~ms}$ relative to the onset of the Noun1. A $200 \mathrm{~ms}$ pre-Noun1 baseline correction was applied for each epoch. We also performed an ERP analysis at the second noun following the verb, but since we found no significant effects when comparing conditions we report only the analysis performed at the Noun1. ERPs were calculated by averaging the EEG segments over all trials in each condition, for each participant, and then computing condition-specific grand averages over all participants. We used a region of interest (ROI) approach for the statistical comparisons, averaging data from single electrodes at three ROIs: Anterior (Fp1, Fpz, Fp2, AF3, AFz, AF4, F7, F5, F3, F1, Fz, F2, F4, F6, F8); Central (FC5, FC3, FCz, FC4, FC6, C5, C3, Cz, C4, C6, CP5, CP3, CPz, CP4, CP6); and Posterior (P5, P3, P1, Pz, P2, P4, P6, PO7, PO5, PO3, POz, PO4, PO6, PO8, $\mathrm{Oz}$ ). The ROI was included as a fixed within-group factor.

The choice of time windows for the statistical analysis was based on visual inspection of the grand average waveforms and on theoretical considerations. We selected two time windows for the Noun1, in which ERP components previously described in the literature have been found: a positive peak over anterior and central electrodes between 150 and $350 \mathrm{~ms}$ (P300-like); and a negative-going peak between 350 and $550 \mathrm{~ms}$ (N400).

For each time window a 2 Congruence (Incongruent, Congruent) $\times 2$ Sentence Type (Action, Perceptive) $\times 3$ ROI (Anterior, Central, Posterior) ANOVA was performed, using the mean amplitude in the window as a dependent variable. Significant interactions were clarified by breaking them down into simple effects. Greenhouse-Geisser correction was used where appropriate (when Mauchly's Test for sphericity was significant). 


\section{Time-frequency (TF) analysis}

The time-frequency representations of the single trial data (epochs from $-2000 \mathrm{~ms}$ to $4000 \mathrm{~ms}$ relative to the onset of the verb) were computed using a sliding window with a Hanning taper. Time-frequency representations (TFRs) were constructed for a low frequency range $(2-30 \mathrm{~Hz})$, using $2.5-\mathrm{Hz}$ frequency-smoothing and $400 \mathrm{~ms}$ timesmoothing. Effectively, in terms of temporal resolution, this means that any given time point in the resulting TFR is an average of the time points ranging from $200 \mathrm{~ms}$ before to $200 \mathrm{~ms}$ after that time point. Power changes were computed in frequency steps of $1 \mathrm{~Hz}$ and time steps of $10 \mathrm{~ms}$. Averaged TFRs were computed for each condition and each participant separately by averaging the power over all trials in every time-frequency bin for each individual electrode. The resulting participant-averaged power changes in the post-stimulus interval were expressed as an absolute change from the baseline interval (-500 to $0 \mathrm{~ms}$ relative to verb onset). Differences between conditions were tested for statistical significance using a cluster-based random permutation procedure to identify consistent differences between conditions in time-frequency clusters. This approach allows us to control for multiple comparisons based on physiologically plausible assumptions (Maris and Oostenveld, 2007). First, t-tests were computed and thresholded ( $\alpha=$ $5 \%$ ) for the difference between conditions for frequency-time pairs at the electrodes of interest (see below). Next, significant frequency-time pairs were clustered in frequency and time if they showed the same direction of effect. To assess statistical significance of each cluster, a cluster level test statistic was calculated by computing the sum of all t-statistics in the cluster. The significance of each cluster-level statistic was estimated by comparing the cluster statistic to a permutation distribution derived from the data, with cluster statistics falling in the highest or lowest 2.5th percentile considered significant. The permutation distribution is the distribution of the test statistic under the null hypothesis that the distributions of the two conditions are identical. The permutation distribution was obtained by randomly permuting the data 1000 times.

We selected two ROIs: the fronto-central area and the parietooccipital area. The fronto-central ROI was obtained by averaging the electrodes over the sensoriomotor cortex (FC1, FC2, C3, Cz, C4), applying the cluster-based random permutation procedure according to the topography of motor related mu oscillatory rhythms from previous studies (Cochin et al., 1998; McFarland et al., 2000b; Muthukumaraswamy et al., 2004; Oberman et al., 2005; Quin and He, 2005). This ROI was selected to contrast CA vs. ABS, and CA vs. CP to verify whether action language differentially modulates $\mathrm{mu} /$ beta rhythms compared with abstract and perceptive language respectively. We also contrasted in this ROI CP vs. ABS to explore the possibility that perceptive language also modulates rhythmic activity of mu/beta, or perhaps shows effects in some other frequency range. Finally, we tested for possible congruence effects, by comparing IA vs. CA, and IP vs. CP.

The second ROI was obtained by averaging five parieto-occipital electrodes (PO7, 01, Pz, O2, and PO8), applying the cluster-based random permutation procedure, with the aim of exploring possible differences in alpha rhythms related to visual or attentional processing (Moreno et al., 2013). The same contrasts tested in the fronto-central ROI, were also explored in the parieto-occipital ROI, although we predict that in this case action-related effects will not be obtained.

\section{Source analysis}

The purpose of the source analysis was to identify the sources of the oscillatory activity for which significant clusters were obtained in the TF analysis. Therefore, the sources were estimated for the following comparisons: CA vs. ABS, CA vs. CP, and IA vs. CA. A beamforming approach was used (Dynamic Imaging of Coherent Sources; DICS). The DICS technique uses adaptive spatial filters to localize power in the entire brain (Gross et al., 2005; Liljestrom et al., 2005). Sources were reconstructed using the frequency and time ranges which characterized the statistically significant clusters from the TF analysis. For the contrast CA-ABS, trials were segmented into two time windows: one extending from 1500 to $2300 \mathrm{~ms}$ relative to verb onset (the time-window of cluster 1 of the TF analysis, approximately corresponding to Noun1), and another from 2600 to $3400 \mathrm{~ms}$ relative to verb onset (the time-window of cluster 2 of the TF analysis, which corresponds to the second noun (Noun2). For the source reconstruction, the data from all electrodes were rereferenced to the average of all electrodes (common average reference). We used a center frequency of $9.6 \mathrm{~Hz}$ with frequency smoothing of $\pm 3.75 \mathrm{~Hz}$. For the contrast $\mathrm{CA}-\mathrm{CP}$, trials were segmented into a time window from 2600 to $3400 \mathrm{~ms}$ relative to verb onset, corresponding to the only significant TF cluster, employing a center frequency of $9.6 \mathrm{~Hz}$ with frequency smoothing of $\pm 2.5 \mathrm{~Hz}$. Finally, for the contrast IA-CA, trials were segmented into a time window from 2000 to 2500 ms relative to verb onset, and a center frequency of $16 \mathrm{~Hz}$ with frequency smoothing of $\pm 4 \mathrm{~Hz}$ was used. For each comparison a frequency analysis was carried out using the multitaper method based on discrete prolate spheroidal sequences (Slepian sequences) (Mitra and Pesaran, 1999).

Standard electrode locations were used in the source reconstruction for all participants, and the electrodes were aligned to a volume conduction model. The volume conduction model was calculated using the boundary element method using a standard MNI/SPM brain (Montreal Neurological Institute (MNI), Montreal, Quebec, Canada; http://www. bic.mni.mcgill.ca/brainweb) (Oostenveld et al., 2001). This standard volume conduction model was used for all participants. Spatial filters were computed (over all participants) at a discrete grid of source points with $1 \mathrm{~cm}$ resolution using all the trials from all three conditions. Next, source power values were computed using this common filter for each participant in each condition. Power values were then logtransformed, which makes the power values more normally distributed and reduces the influence of outliers. Grand averaged sources were calculated by averaging the individual sources across participants and conditions. Contrasts were defined as the absolute difference in power between the two conditions. For the evaluation of the differences between conditions for the source reconstructed power data, paired t-tests were computed at each source location, and then neighboring source-locations with significant (uncorrected) t-values were clustered. Then, the sum of the t-values in each cluster was calculated, and we selected the cluster with the largest sum of the t-values.

\section{Results}

\section{Behavioral results}

For the catch trials, participants judged the congruent sentences to be more coherent than the incongruent sentences, indicating that they were paying attention to the meaning of the sentences. Thus the average percentage of "Yes" responses and the associated standard deviations for the coherence judgments were: $\mathrm{CA}: \mathrm{M}=$ 96.7, $\mathrm{SD}=0.07$; IA: $\mathrm{M}=3.3, \mathrm{SD}=0.12 ; \mathrm{CP}: \mathrm{M}=95, \mathrm{SD}=0.08$, and IP: $M=5, S D=0.13$. The contrasts between congruent/incongruent pairs for both action and perceptive conditions were significant: CA $>$ IA $(\mathrm{t}(23)=2.89, p<.01)$, and CP $>$ IP $(t(23)=2.29$, $p<.05)$.

\section{ERP analyses}

For the sake of brevity, only statistically significant effects in the ERPs will be reported.

\section{0-350 ms window (P300)}

The most important result was a significant Sentence Type $\times$ Congruence interaction: $\mathrm{F}(1,23)=5.51$, MSE $=1.73, p<.03$. The follow up pairwise comparisons revealed that the incongruent condition was 


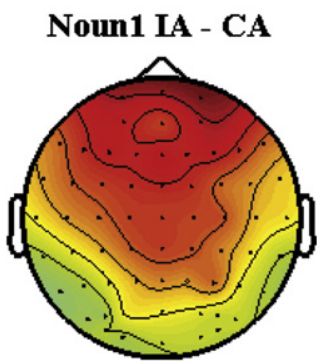

$150-350 \mathrm{~ms}$
Noun1 LA and CA (Fz)

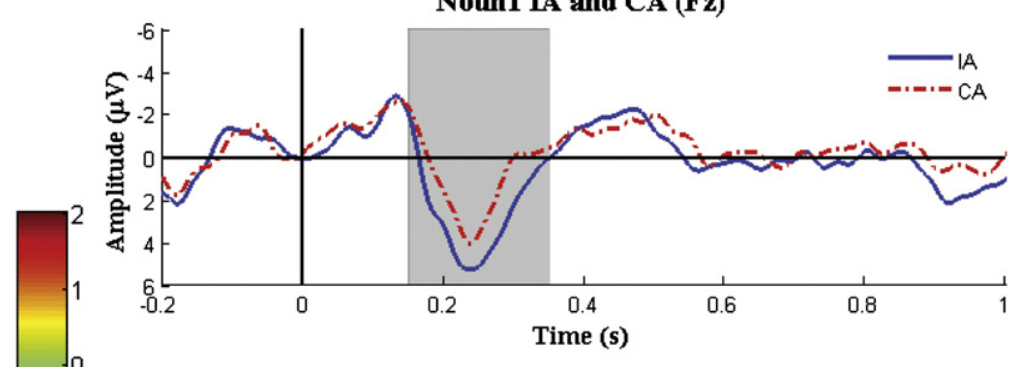

Noun1 LA and CA (Pz)

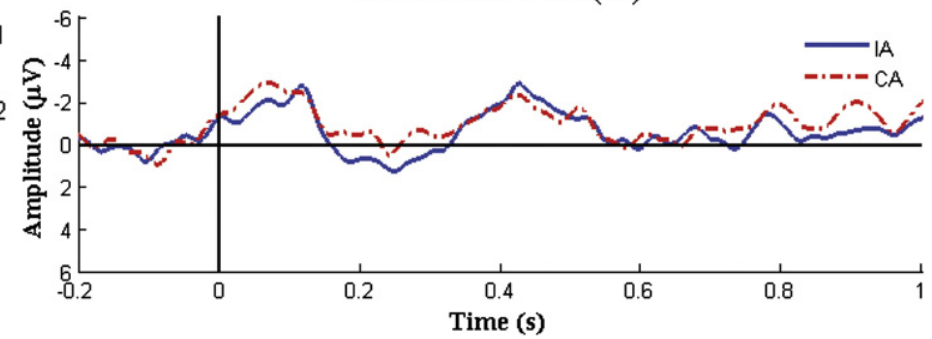

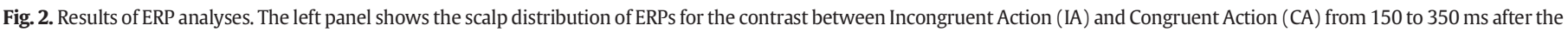

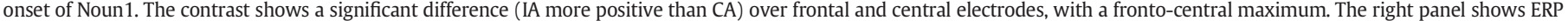

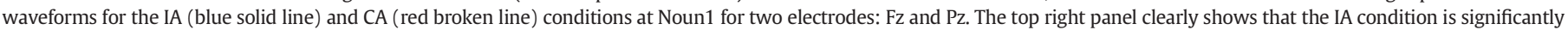

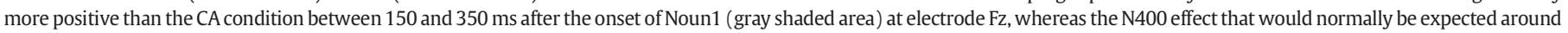
Pz was absent (bottom right).

significantly more positive than the congruent condition for action sentences $(\mathrm{M}=1.05 \mu \mathrm{V}, \mathrm{SE}=0.37$, and $\mathrm{M}=0.10 \mu \mathrm{V}, \mathrm{SE}=0.26$ respectively; $t(23)=3.39, p<.0044)$, but not for perceptive sentences $(\mathrm{M}=$ $0.70 \mu \mathrm{V}, \mathrm{SE}=0.3$, and $\mathrm{M}=0.48 \mu \mathrm{V}, \mathrm{SE}=0.24$ respectively; $t(23)=$ $0.902, p=.38)$. Fig. 2 shows the grand average ERP waveforms for the IA and the CA conditions for a representative electrode Fz (top right). The same figure (left) shows the scalp topography for the difference in voltage between IA and CA in the time window between 150 and $350 \mathrm{~ms}$.

\section{0-550 ms window (N400)}

A significant main effect of Sentence type was observed: $F(1,23)=$ 4.458, $\mathrm{MSE}=1.443, p<.047$ (CA: $\mathrm{M}=-1.182, \mathrm{SE}=0.164 ; \mathrm{CP}$ : $\mathrm{M}=-1.481, \mathrm{SE}=0.148$ ), but neither the main effect of Congruence nor its interaction with other variables were significant. In other words, no N400 effect associated with Congruence was found (Fig. 2, bottom right panel).

\section{TF and source analyses}

Only comparisons that reached statistical significance in the TF analysis will be reported. The CA-ABS, and the CA-CP comparisons produced significant effects at the selected fronto-central region (FC1, $\mathrm{FC} 2, \mathrm{C} 3, \mathrm{Cz}$, and $\mathrm{C} 4$ ), whereas the IA-CA comparison produced significant effects at the parieto-occipital region (PO7, 01, Pz, O2, and PO8). Sources of the oscillatory activity for significant clusters of each comparison obtained in the TF analysis are also reported. One potential concern might be that our sensor-level oscillatory analysis compared baseline corrected power values between the different conditions, while our source-level oscillatory analysis compared power values that were not baseline corrected. This could mean that differences in baseline power might (at least partially) drive the effects for our sensor-level analysis, making them less comparable to the effects found at the level of oscillatory sources. To address this issue we used the same random permutation testing procedure used in our main analyses to test for power differences in the baseline intervals used for the sensor-level analysis. We did not find significant differences in baseline power when comparing any conditions, either over the fronto-central ROI or the parieto-occipital ROI. We thus conclude that our mu effects for the sensor-level analysis are not driven by differences in the level of baseline power between conditions, and these results highlight the same oscillatory effects found in our source-level analysis. These results are presented in detail below.

\section{CA-ABS comparison}

At the fronto-central region the individual power spectra for the CA condition showed a decrease in power in the mu frequency range starting around $200 \mathrm{~ms}$ after the verb onset, and extending across the rest of the sentence, including Noun1 and Noun2 (top left part of Fig. 3A). By contrast, for the ABS condition a small decrease in power in the mu frequency range was only present between approximately 300 and $700 \mathrm{~ms}$ after the verb onset, which was followed by an increase in power in this range starting around the onset of the Noun1 (1300 ms) and lasting until the end of the Noun2 (3300 ms), as shown in the top right part of Fig. 3A. The comparison of the spectral power indicated a strong negative difference between the CA and the ABS conditions in the $6-13 \mathrm{~Hz}$ frequency range across the whole sentence (see bottom left part of Fig. 3A). Finally, the statistical contrast CA-ABS showed two significant negative clusters in the frequency range of $6-13 \mathrm{~Hz}$, the first one extending from 1500 to $2300 \mathrm{~ms}(p=.01)$, and the second one extending from 2600 to $3400 \mathrm{~ms}(p=.01)$ after the verb onset. Notice that the first cluster starts about $200 \mathrm{~ms}$ after the Noun 1 onset, and the second cluster occurs around the Noun2 onset (Fig. 3A, bottom right). Fig. 3B, shows the scalp distribution of the two significant negative clusters for the mu frequency range. The difference showed a fronto-central distribution with a central maximum for both clusters.

The cluster-level randomization test showed no significant differences over posterior electrodes for this comparison, indicating that the effects cannot be confused with posterior alpha.

Fig. $3 \mathrm{C}$ shows the source reconstruction of oscillatory activity in the two significant negative clusters obtained in the TF analysis for the CAABS comparison. The strongest locus of the source activity of cluster 1 , using the time window $1500-2300 \mathrm{~ms}$ relative to verb onset, and the frequency range $9.6 \pm 3.75 \mathrm{~Hz}$, was BA 6 around the right superior frontal gyrus ( $x=8, y=7, z=69$; Fig. $3 C$, top part). The strongest locus of the source activity of cluster 2, using the time-window 2600-3400 ms relative to verb onset and the same frequency range, was BA 39 around the left superior temporal gyrus $(x=-55, y=-62, z=23)$. 


\section{Contrast CA - ABS}

\section{A) TF representations}
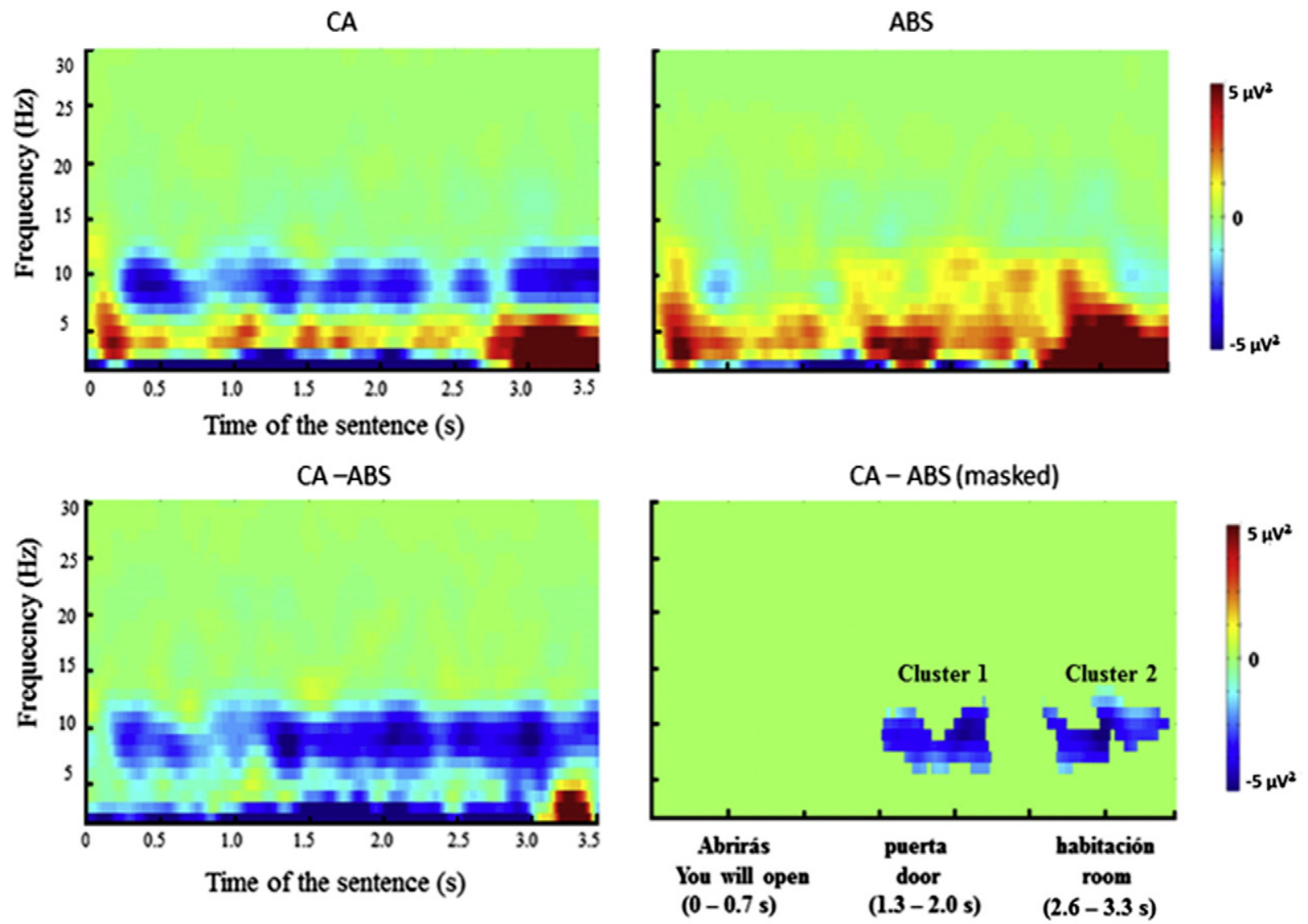

B) Scalp topography

Cluster 1

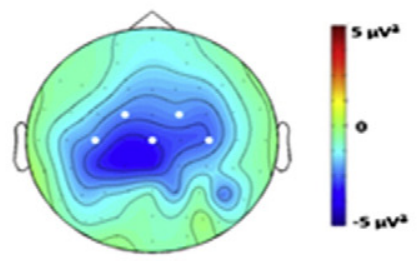

$6-13 \mathrm{~Hz}, 1.5-2.3 \mathrm{~s}$
C) Source analysis

Cluster 1
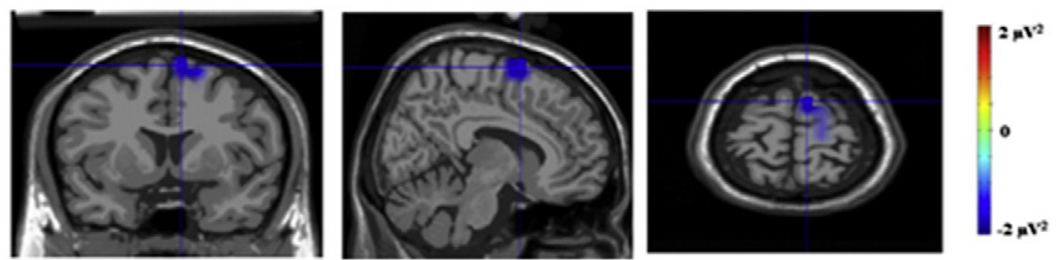

$\mathrm{CA}<\mathrm{ABS}: \mathrm{x}=8, \mathrm{y}=7, z=69$

Cluster 2
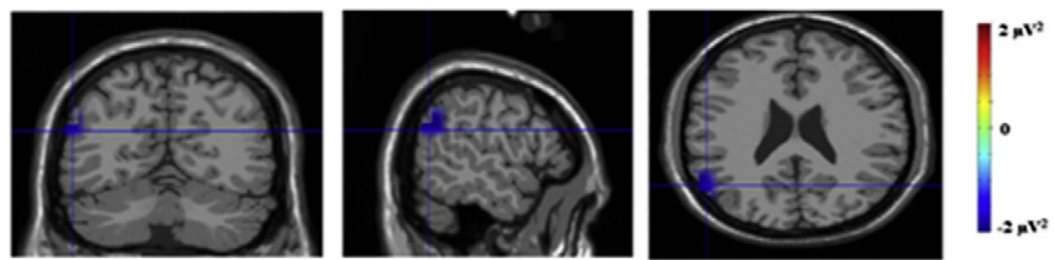

$6-13 \mathrm{~Hz}, 2.6-3.4 \mathrm{~s}$

$\mathrm{CA}<\mathrm{ABS}: \mathrm{x}=-55, \mathrm{y}=-62, \mathrm{z}=23$ 


\section{CA-CP comparison}

At the fronto-central region a decrease in mu power was present in the CA condition, starting around $200 \mathrm{~ms}$ after the verb onset and continuing across the rest of the sentence, including the Noun 1 and the Noun2, as the top left part of Fig. 4A illustrates. In the same frequency range a decrease in power was present in the $\mathrm{CP}$ condition, extending from 400 to $2300 \mathrm{~ms}$ after the verb onset. This was followed by an increase in power from about $2300 \mathrm{~ms}$ to $2500 \mathrm{~ms}$ after verb onset, before another decrease in power starting around the onset of the Noun2 (2600 ms), and lasting until the end of the sentence (Fig. 4A, top right). Comparison of the power spectra indicated a negative difference between conditions in the mu frequency range extending across the entire sentence, except for small positive differences between about 0 and $200 \mathrm{~ms}$ and between 700 and $900 \mathrm{~ms}$ relative to verb onset (Fig. 4A, bottom left). The cluster-level randomization testing revealed a significant negative cluster for the contrast $\mathrm{CA}-\mathrm{CP}$, in the mu frequency range $(8-11 \mathrm{~Hz})$ extending approximately from 2800 to $3400 \mathrm{~ms}$ after the verb onset $(p<.05)$. Notice that this cluster starts approximately $200 \mathrm{~ms}$ after the Noun 2 onset (Fig. 4A, bottom right). Fig. $4 \mathrm{~B}$, shows the scalp distribution of the time-frequency data collapsed over frequencies from 8 to $11 \mathrm{~Hz}$ and times from 2800 to $3400 \mathrm{~ms}$ for the cluster found for the CA-CP comparison. The scalp distribution shows a negative difference around the fronto-central electrodes (slightly biased towards the right hemisphere). The cluster-level randomization test showed no significant differences over posterior electrodes for this comparison.

Fig. 4C shows the strongest locus of the source of the significant mu power difference, for the frequency range $9.6 \pm 2.5 \mathrm{~Hz}$, for the CA-CP comparison in a time-window from 2600 to $3400 \mathrm{~ms}$ after verb onset. The locus of the effect was strongest in BA 6 around the superior frontal gyrus $(\mathrm{x}=-1, \mathrm{y}=24, \mathrm{z}=61)$.

\section{IA-CA comparison}

At the parieto-occipital sites the IA condition exhibited a decrease in power in the low beta range $(13-20 \mathrm{~Hz})$, starting with the verb onset, and extending across the entire sentence (see top left part of Fig. $5 \mathrm{~A}$ ). In the same frequency range a decrease in power was present in the CA condition starting around $200 \mathrm{~ms}$ after the onset of the verb and extending across the entire sentence, except for a small increase in power between 2200 and $2500 \mathrm{~ms}$ (see top right part of Fig. 5A). The comparison of power spectra in the IA and CA conditions revealed a negative difference in the low beta frequency range $(13-16 \mathrm{~Hz})$ starting around the onset of the verb and extending across the rest of the sentence, and a positive difference in beta power $(16-20 \mathrm{~Hz})$ from around 400 to $2200 \mathrm{~ms}$ after the verb onset. This positive difference in power was followed by a negative power difference in the same frequency range between 2200 and $2500 \mathrm{~ms}$, and then another positive difference lasting to the end of the sentence. The cluster-level randomization test for the contrast IA-CA showed a significant negative cluster in the low beta frequency range $(13-20 \mathrm{~Hz})$ extending from 2200 to $2500 \mathrm{~ms}$ after the verb onset $(p<.05)$, as Fig. $5 \mathrm{~A}$, bottom right illustrates. Fig. $5 B$, shows the scalp distribution of the time-frequency data for the IA-CA comparison in the beta frequency range $(13-20 \mathrm{~Hz})$ for the 2200 to $2500 \mathrm{~ms}$ after verb onset time window. The cluster-level randomization test showed no significant differences over fronto-central electrodes for this comparison.

Fig. 5C shows the strongest locus of the source of the beta power difference for the IA-CA contrast. Source reconstruction for this comparison was computed in the $16 \pm 4 \mathrm{~Hz}$ frequency range and the 2000 to $2500 \mathrm{~ms}$ time-window relative to verb onset. In this case, the locus of the effect was strongest in BA 19 around the left middle temporal gyrus ( $\mathrm{x}=-49, \mathrm{y}=-80, \mathrm{z}=8$ ), which corresponds to the extrastriate body area (EBA).

\section{Discussion}

This study analyzed whether motor-related brain activity is specifically and distinctively involved in the comprehension of actionlanguage. Unlike other studies that focused on the functional neuroanatomy of motor-related cortical activity (e.g., fMRI experiments), this study is primarily concerned with the brain dynamics underlying the comprehension of action sentences. With this aim, EEG data were collected while participants read action sentences, perceptive sentences, and abstract sentences. These EEG data were then analyzed using time-frequency analysis, ERP analysis and source estimation.

In brief, these are the most relevant results. First, we found that mu rhythms were suppressed by congruent action sentences but not by abstract sentences (Fig. 3A). Second, mu suppression was also significantly larger in congruent action language than in congruent perceptive language, although some mu suppression can also be observed in the latter (Fig. 4A). Third, the source reconstruction confirmed that the mu suppression for the congruent action language was strongest in the premotor cortex (Figs. 3C, and Fig. 4C). Fourth, in congruent action sentences mu suppression reached a maximum at the two nouns following the action verb, as indicated by the temporal locus of the significant clusters in the TF analysis (Fig. 3A). This suggests that motor activation is associated with integrative processes across multiple words in action sentences, rather than being a purely lexical feature of action verbs. Finally, congruent and incongruent action sentences differentially modulate beta rhythms at posterior sites in the contrast IA vs. CA (Fig. 5A). The source estimation suggests that the locus of this beta activity is the visual cortex; specifically the extrastriate body area (EBA), rather than the motor cortex (Fig. 5C).

\section{Mu suppression in action language}

Using abstract language as a contrasting condition for exploring motor related activity in action language is a common strategy in neuroimaging studies (Aziz-Zadeh et al., 2006; Tettamanti et al., 2005), and has also been used in a previous study of mu rhythm modulation (Moreno et al., 2013). In the latter study we also found strong evidence, based on mu suppression, that action language activates motor networks, whereas abstract language does not. However, here the goal was to employ a more fine-grained test of motor-related processing by contrasting action language with concrete perceptive language, in order to eliminate the possibility that the previously observed effects were a result of a difference in concreteness, rather than being related to action language. Further, given the fact that perception and action are closely related processes (e.g., Pulvermüller, 1996; Rizzolatti and Arbib, 1998; Rizzolatti and Craighero, 2004; Rizzolatti and Luppino, 2001), it is possible that action language and perceptive language share common neuronal networks, and thus modulate mu rhythms in a similar way. Our results suggest that mu suppression located at fronto-central sites is modulated mainly by congruent action language rather than congruent perceptive or abstract language. We found strong and differential mu suppression for the congruent action vs abstract

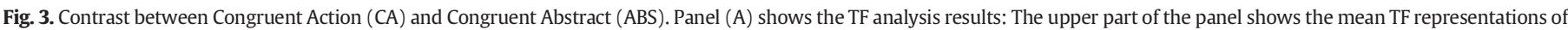

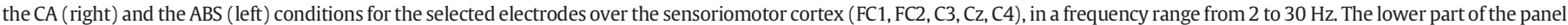

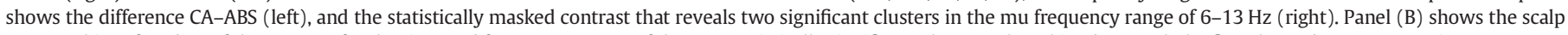

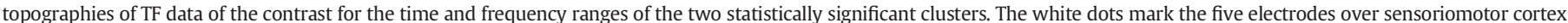

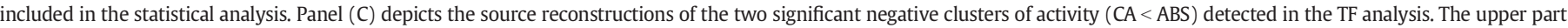

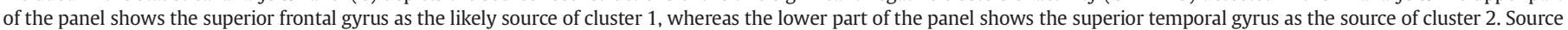
activation is projected onto a standard brain (MNI/SPM). 


\section{Contrast CA - CP}

\section{A) TF representations}

CA

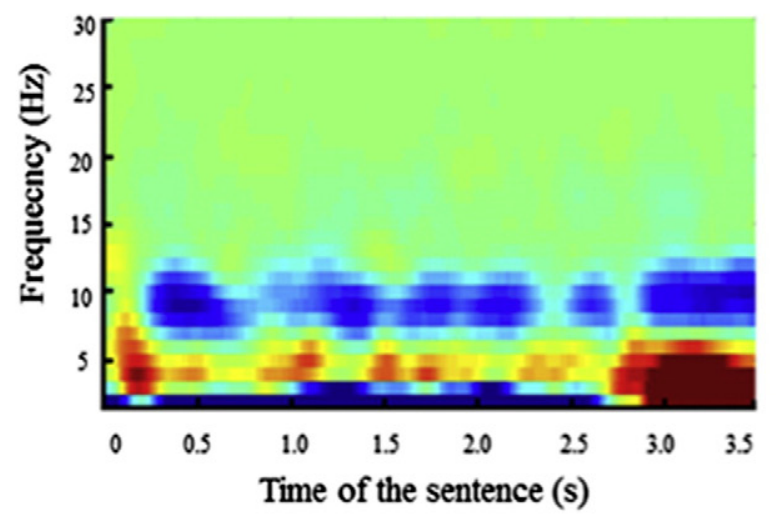

$\mathrm{CA}-\mathrm{CP}$

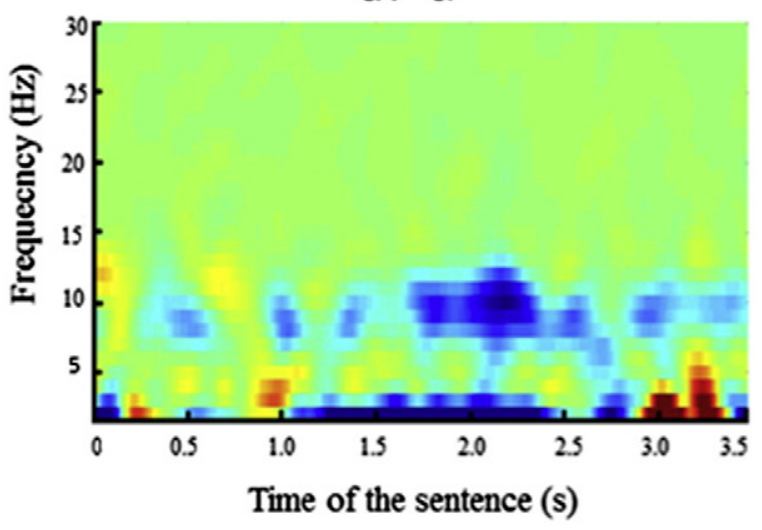

\section{B) Scalp topography}

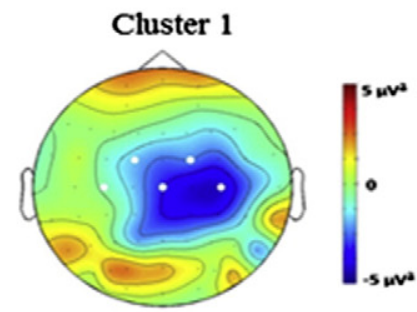

$8-11 \mathrm{~Hz}, 2.8-3.4 \mathrm{~s}$

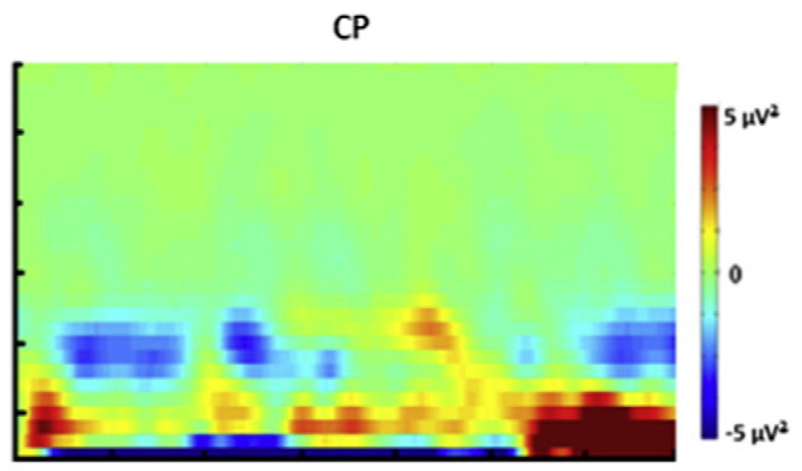

$\mathrm{CA}-\mathrm{CP}$ (masked)

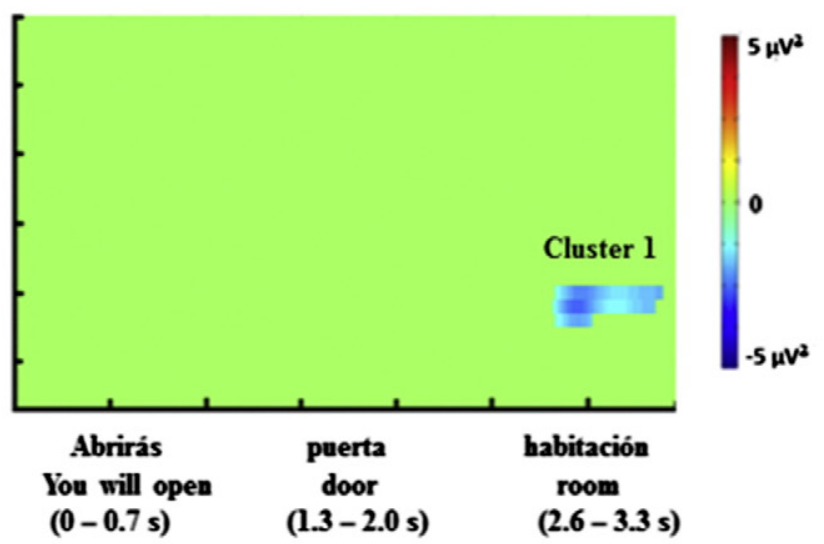

C) Source analysis

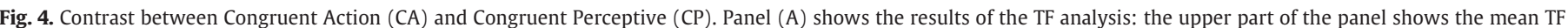

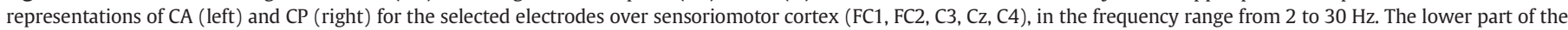

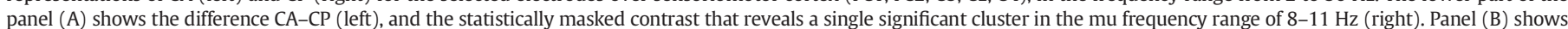

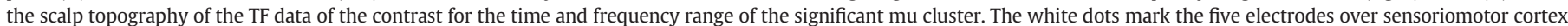

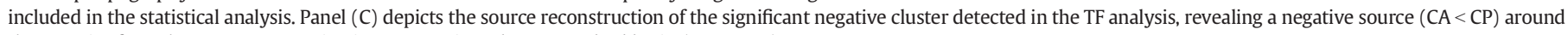
the superior frontal gyrus. Source activations are projected on a standard brain (MNI/SPM).

contrast. Congruent action sentences also elicited larger mu suppression than congruent perceptive sentences, although it should be noted that significant differences in mu suppression are delayed in time, as we will comment on later. This might suggest that some motor-related activity is present during the comprehension of congruent perceptive sentences. However, this motor-related activity is not strong enough as to produce statistical effects in the comparison between congruent perceptive sentences and abstract sentences; thus a small frontocentral negative cluster observed between congruent perceptive and congruent abstract sentences (not shown in the figures) did not reach even marginal statistical significance ( $p=.39$ ). Overall then, these findings support the argument that mu suppression, which can be taken as an index of motor cortical activation, is being observed quite selectively for congruent action language.

The mu modulation associated with congruent action language was strongest in the dorsal premotor area (BA 6), while participants were reading the first noun (contrast congruent action - congruent abstract, Fig. 3C) or the second noun (contrast congruent action - congruent perceptive, Fig. 4C). These findings directly support the notion that understanding congruent action language elicits activation in the 


\section{Contrast IA - CA}

\section{A) TF representations}

IA

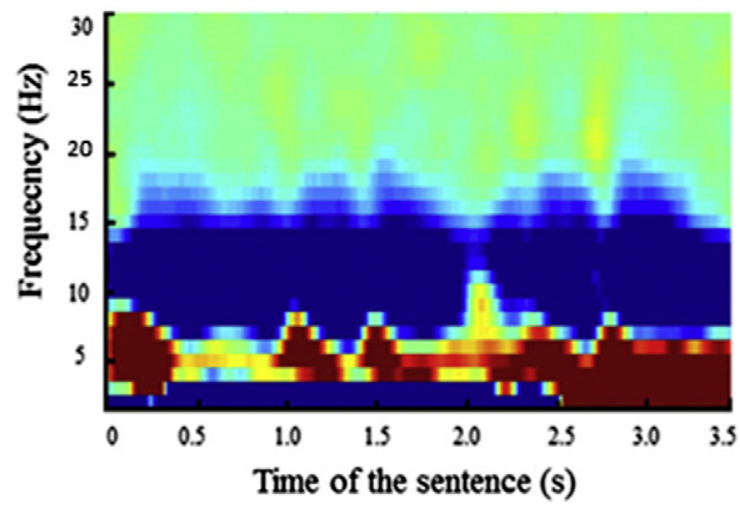

IA-CA

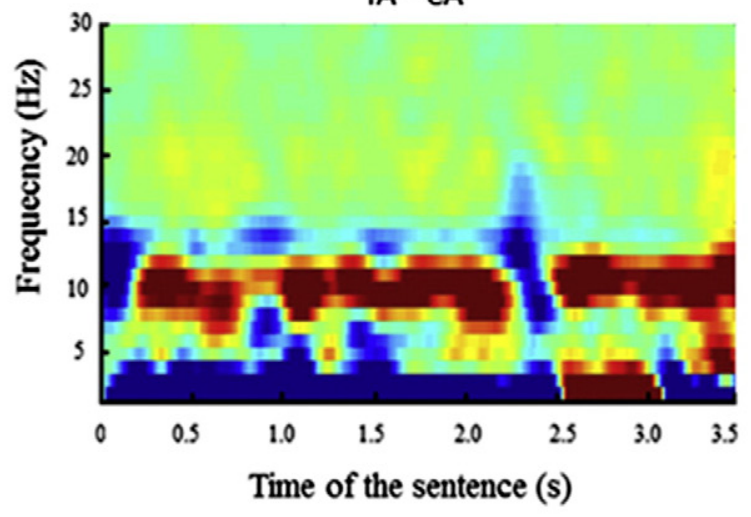

CA

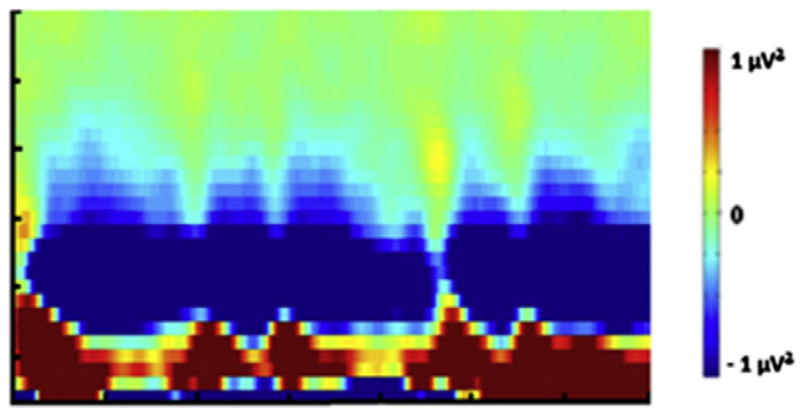

IA-CA (masked)

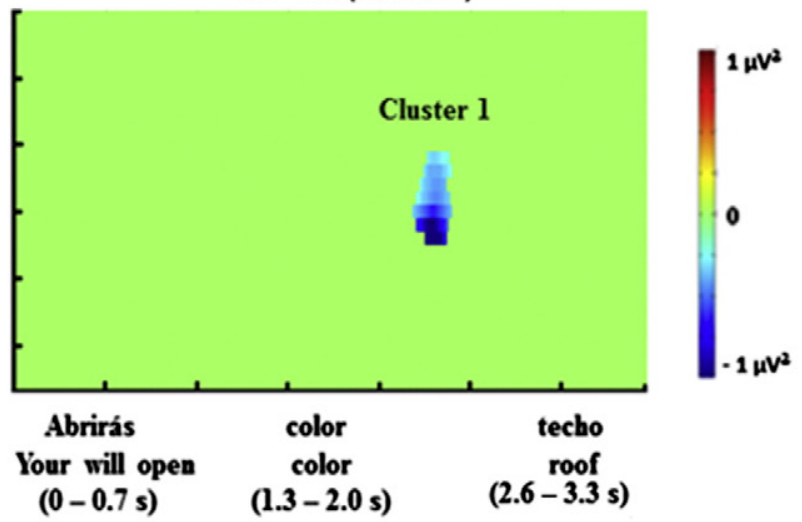

B) Scalp topography

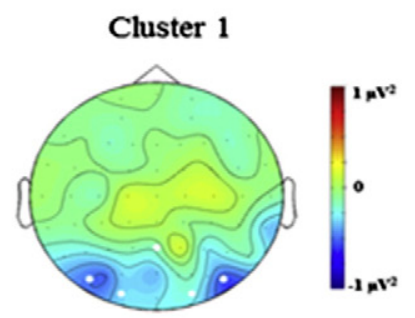

$13-20 \mathrm{~Hz}, 2.2-2.5 \mathrm{~s}$
C) Source analysis
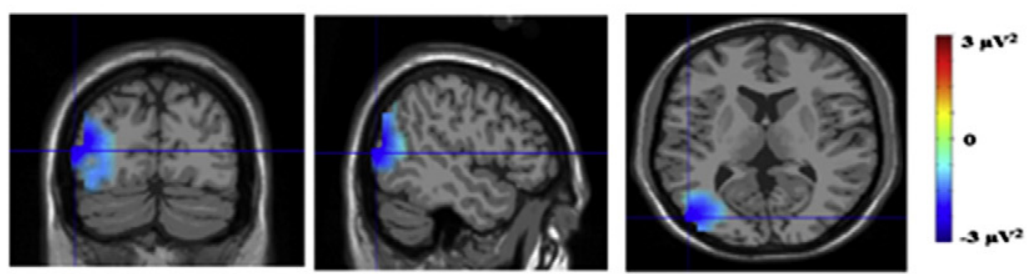

IA $<$ CA: $x=-49, y=-80, z=8$

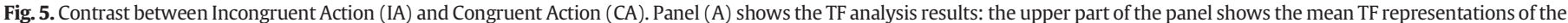

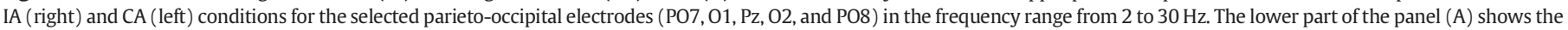

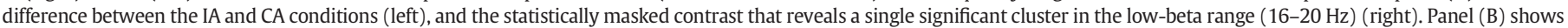

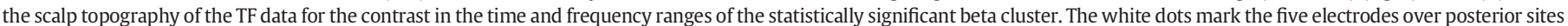

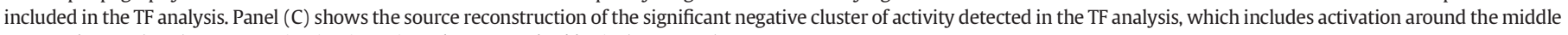
temporal gyrus (EBA). Source activation is projected on a standard brain (MNI/SPM).

premotor cortex (PM). The fact that PM was found in the source reconstruction of both comparisons is in line with previous neuroimaging studies that have consistently shown the involvement of the PM cortex during the processing of action language (Papeo et al., 2009; Pulvermüller et al., 1999; Raposo et al., 2009; Schuil et al., 2013; Tomasino et al., 2008; Willems et al., 2010). In addition, other studies have shown that the dorsal PM is particularly activated during the preparation of movements (Godschalk et al., 1985; Halsband and Freund,
1990; Passingham, 1988), which fits well with the fact that sentences in this study were constructed in future tense (Candidi et al., 2010). The differential suppression of mu was also observed in the superior temporal gyrus (BA 39), while participants were reading the final word of congruent action sentences (contrast congruent action - abstract; Fig. 3C). As with most regions in the cortex, the superior temporal gyrus is involved in different functional networks, including the motor neural network. Thus, it has been related to higher-order 
processing of willed actions (Frith et al., 1991), and hand actions (Buccino et al., 2001). Given that our action sentences always referred to hand actions we can speculate that the identified source in the superior temporal gyrus is also related to higher-order motor processing.

\section{Time course of mu suppression}

As we mentioned in the Introduction, one goal of this study was to investigate the temporal dynamics of motor-related activation during the comprehension of action sentences. Notice that unlike in previous studies (van Elk et al., 2010) we examined the role of integrative processing across the whole sentence rather than only at the verb. This was possible because the action sentences in this study were constructed in such a way that the action was not yet fully specified at the verb, but was completed by the nouns that followed the verb. In this study the TF analysis clearly showed that statistically significant differences in mu suppression first appeared while reading the first noun (contrast congruent action vs congruent abstract), or while reading the second noun (contrast congruent action vs congruent perceptive), indicating that the motor-related activation was quite dependent on the integration of lexical semantics across the sentence. Thus, although there was some mu suppression throughout the whole coherent action sentence, we observed maximal mu suppression only when the action verb and the noun converged onto a concrete action. These results are in line with some embodiment theories, which claim that motor-related activation is context-dependent rather than purely lexical (Aziz-Zadeh et al., 2006; Boulenger et al., 2009; Schuil et al., 2013; Taylor et al., 2008). The fact that mu suppression effects were delayed until the second noun in the congruent action vs congruent perceptive contrast could indicate that understanding congruent perceptive sentences also elicits activation of motor assemblies, and only at the end of the congruent action sentence enough motor activation has been accumulated (mu suppression) to make the difference between the two conditions emerge sufficiently strongly to be detectable at the scalp. Nonetheless, the motor resonance associated with congruent perceptive sentences, if any, would be weak at best, because as mentioned before the contrast between the congruent perceptive vs congruent abstract conditions did not yield a significant effect.

We cannot rule out the notion that motor-related activity due to lexical level processing may occur under certain circumstances. Most of the action verbs employed here corresponded to rather generic actions, like "to get", "to open", or "to cut" which could be realized as substantially different motor programs depending on the particular object to which they refer. This may have forced the reader to continue reading to get enough information from the incoming words before being able to 'simulate' the action. However, if the verb had been associated with relatively specific motor programs, like "to caress", "to write" or "to punch", then the verb alone may have been sufficient to elicit motor activation.

\section{Congruence effects in action language}

The behavioral data show that participants judged congruent sentences as more coherent than incongruent sentences. Therefore, we can assume that they processed the sentences' meaning as we expected with our materials. The ERP analysis also showed congruence effects for action sentences. Specifically, the incongruent action elicited a more positive wave than the congruent action in the 150-350 ms time window after the onset of the first noun. Based on the latency and shape of the waveform, as well as the fronto-central scalp distribution, we can identify this component as a P3a (Polich, 2007). The P3a is typically elicited by novel or less frequent stimuli in oddball tasks (see review by Polich, 2007), or in motor (button-press) and cognitive (counting) go/no go tasks (Bokura et al., 2001; Bruin and Wijers, 2002; Smith et al., 2008). However, our task here cannot be easily compared with any of these paradigms. Congruent and incongruent action sentences were equally probable (50\%), and in most trials participants just read the sentences passively. Only in about $12 \%$ of trials were participants asked to make a speeded coherence judgment (catch trials), but the prompt for response was presented after collecting the EEG in the whole sentence. But the P3a is also elicited when the stimuli attract attention without the task necessarily making one type of stimulus more salient than the others (Reinvang, 1999). In the present study there was a more conspicuous P3a in incongruent rather than congruent action sentences (Fig. 2), indicating that it was incongruent that showed a larger peak and were more attention attracting. Given the fact that the congruence effect requires the combination of the verb and the Noun1, our P3a effect indicates that participants (or at least their ERPs) are sensitive to integration processes across the sentences.

We did not find any N400 effect associated with congruence. This might seem strange because, the behavioral data demonstrated that readers were sensitive to the semantic congruence of sentences, judging the incongruent less coherent than the congruent versions. A possible explanation for this lack of standard N400 effects is that our manipulation of congruence was concerned with the feasibility of actions and perceptions, in accordance with our norming study, rather than with the cloze probability of the nouns. To support this explanation, we checked a posteriori the cloze probability of the verb-nouns pairs selected for our congruent and incongruent sentences. Twenty-seven new participants were presented the action and the perceptive verbs and were asked to generate a noun that could continue the sentence. The cloze-probability of nouns was calculated by taking the probability that a group of individuals would complete a particular sentence fragment with that noun in an offline sentence completion task. Cloze probability is typically used as a measure of the degree of semantic fit between a word and it's preceding sentence context (DeLong et al., 2005; Kutas and Hillyard, 1984). It turned out that all our incongruent sentences had a cloze probability of zero, whereas the congruent sentences had an average cloze probability close to zero (congruent action sentences $=0.12$; congruent perceptive sentences $=0.07$ ). This means that the sentences used in the congruent conditions were not strongly constraining in terms of lexical-semantic expectancy, and this likely resulted in the lack of N400 effect for the IA-CA (Fig. 2) and IP-CP (not shown) comparisons. However, this lack of N400 effects is not particularly relevant in this study. Moreover, it is a guarantee that our ERP congruence effects for action sentences (P3a) cannot be confounded with the effect of lexical-semantic constraints in the sentence.

The most important effect of congruence was observed in the TF analysis. Differential suppression in the low-beta range $(13-20 \mathrm{~Hz}$ ), with a posterior distribution, was found in the incongruent action condition compared to the congruent action condition. Let us consider three possible explanations. First, beta rhythms, together with mu rhythms, are typically considered to be electrophysiological markers of motor-related activity in the brain. Many studies have found both mu and beta suppression when people perform actions, observe actions or imagine themselves performing actions (Jurkiewicz et al., 2006; McFarland et al., 2000a, 2000b; Neuper et al., 2006; Orgs et al., 2008; Pfurtscheller and Lopes da Silva, 1999; Pfurtscheller et al., 2006; Pineda, 2005). Suppression of beta rhythms has also been reported when people understand action language compared to abstract language (Moreno et al., 2013), or action language referring to animals compared to action language referring to humans (van Elk et al., 2010). However, we can rule out that our beta effects are motoric, because the observed modulation occurs over posterior electrodes rather than fronto-central electrodes. Moreover, we did not find differential modulation of beta between congruent action and congruent abstract conditions or between congruent action and congruent perceptive conditions. Second, beta could be considered to be a general signature of incongruence during sentence binding processes (Wang et al., 2012; Weiss and Mueller, 2012). This fits well with the fact that beta suppression was larger in the incongruent action than in congruent action sentences. Also, the significant beta difference in these two 
conditions was observed in a very late time window (2200 to $2500 \mathrm{~ms}$ after the verb onset), a position in the sentence at which the verb and the first noun should already have been integrated, and at which the reader might be most sensitive to the incongruity. However, we did not find a similar beta modulation between the incongruent and congruent perceptive conditions, and thus the "incongruence signature" hypothesis seems unlikely. Finally, the sources of beta could provide a third interpretation of our results. Suppression of beta was localized in coordinates compatible with the extrastriate body area (EBA), which has been related in the literature to visual processing of individual body parts and body motions. Some neuroimaging studies have found that action language could also activate EBA in addition to motor and premotor regions, as another complementary neural assembly for action processing (Rueschemeyer et al., 2010; Urgesi et al., 2007; De Vega et al., 2014; Weiner and Grill-Spector, 2011). In this study, it seems that understanding incongruent action sentences induces readers to utilize an alternative visual network, perhaps to analyze the unusual bodily motion described by the verb. This does not necessarily mean that the activation of motor-related processing is absent in incongruent action sentences. The lack of significant differences in mu suppression over the motor cortex between incongruent and congruent action conditions lets open the possibility that in both cases there is activation in the motor cortex.

\section{Conclusions}

This study provides evidence that the comprehension of action sentences activates the motor cortex on-line, as reflected by mu suppression over fronto-central electrodes. Specifically, the source reconstruction confirmed that this mu modulation occurred in the premotor cortex (supplementary motor area, BA 6). The activation of the motor cortex was significantly larger in action language than in the contrasting conditions, as robust mu suppression was found in action sentences, but not in abstract language. Even concrete perceptive sentences only produced weak mu suppression, which did not significantly differ from abstract language. The time-frequency analysis of mu suppression indicates that the activation of the motor cortex in action sentences requires the semantic integration of words across the sentence, rather than being a purely lexical feature of action verbs.

\section{Acknowledgments}

This research was funded by the Spanish Ministry of Education and Science (Grants PSI2011-28679, and BES-2008-00234), the Canary Agency for Research, Innovation, and Information Society (NEUROCOG Project), and the European Regional Development Funds. We would like to thank Robert Oostenveld for his valuable comments about source analysis reconstruction.

\section{References}

Aziz-Zadeh, L., Wilson, S.M., Rizzolatti, G., Iacoboni, M., 2006. Congruent embodied representations for visually presented actions and linguistic phases describing actions. Curr. Biol. 16 (18), 1818-1823.

Barsalou, L.M., 1999. Perceptual symbol systems. Behav. Brain Sci. 22 (4), 577-660.

Barsalou, L.M., 2008. Grounded cognition. Annu. Rev. Psychol. 59, 617-645.

Barsalou, L.M., Simmons, W.K., Barbey, A.K., Wilson, C.D., 2003. Grounding conceptuals knowledge in modality-specific systems. Trends Cogn. Sci. 7 (2), 84-91.

Bastiaansen, M., Brunia, H.M., 2001. Anticipatory attention: an event-related desynchronization approach. Int. J. Psychophysiol. 43 (1), 91-107.

Bokura, H., Yamaguchi, S., Kobayashi, S., 2001. Electrophysiological correlates for response inhibition in a go/no go task. Clin. Neurophysiol. 112 (12), 2224-2232.

Boulenger, V., Roy, A.C., Paulignan, Y., Déprez, V., Jeannerod, M., Nazir, T.A., 2006. Crosstalk between language processes and overt motor behavior in the first $200 \mathrm{~ms}$ of processing. J. Cogn. Neurosci. 18 (10), 1607-1615.

Boulenger, V., Hauk, O., Pulvermüller, F., 2009. Grasping ideas with the motor system: semantic somatotopy in idiom comprehension. Cereb. Cortex 19 (8), 1905-1914.

Bruin, K.J., Wijers, A.A., 2002. Inhibition, response mode, and stimulus probability: a comparative event-related potential study. Clin. Neurophysiol. 113 (7), 1172-1182.
Brunyé, T.T., Ditman, T., Mahoney, C.R., Augustyn, J.S., Taylor, H.A., 2009. When you and I share perspectives: pronouns modulate perspective-taking during narrative comprehension. Psychol. Sci. 20 (1), 27-32.

Buccino, G., Binkofski, F., Fink, G.R., Fadiga, L., Fogassi, L., Gallese, V., Seitz, R.J., Zilles, K., Rizzolatti, G., Freund, H.J., 2001. Action observation activates premotor and parietal areas in a somatotopic manner: an fMRI study. Eur. J. Neurosci. 13 (2), 400-404.

Buccino, G., Riggio, L., Melli, G., Binkofski, F., Gallese, V., Rizzolatti, G., 2005. Listening to action-related sentences modulates the activity of the motor system: a combined TMS and behavioral study. Cogn. Brain Res. 24 (3), 355-363.

Cacciari, C., Bolognini, N., Senna, I., Pellicciari, M.C., Miniussi, C., Papagno, C., 2011. Literal, fictive and metaphorical motion sentences preserve the motion component of the verb. A TMS study. Brain Lang. 119 (3), 149-157.

Candidi, M., Leone-Fernandez, B., Barber, H.A., Carreiras, M., Aglioti, S.M., 2010. Hands on the future: facilitation of cortico-spinal hand-representation when reading the future tense of hand-related action verbs. Eur. J. Neurosci. 32 (4), 677-683.

Cochin, S., Barthelemy, C., Roux, S., Martineau, J., 1998. Perception of motion and EEG activity in human adults. Electroenceph. Clin. Neurophysiol. 107 (4), 287-295.

Damasio, H., Tranel, D., Grabowski, T., Adolphs, R., Damasio, A., 2004. Neural systems behind word and concept retrieval. Cognition 92 (1-2), 179-229.

Davis, C.J., Perea, M., 2005. BuscaPalabras: a program for deriving orthographic and phonological neighborhood statistics and other psycholinguistic indices in Spanish. Behav. Res. Methods 37 (4), 665-671.

De Vega, M., Glenberg, A., Graesser, A., 2008. Symbols and Embodiment: Debates on Meaning and Cognition. Oxford University Press, New York, pp. 397-440.

De Vega, M., León, I., Hernández, J.A., Valdés, M., Padrón, I., Ferstl, E., 2014. Action Sentences Activate Sensory Motor Regions in the Brain Independently of Their Status of Reality. J. Cogn. Neurosci. 26, 1363-1376.

DeLong, K.A., Urbach, T.P., Kutas, M., 2005. Probabilistic word pre-activation during language comprehension inferred from electrical brain activity. Nat. Neurosci. 8 (8), $1117-1121$

Fargier, R., Paulignan, Y., Boulenger, V., Monaghan, P., Reboul, A., Nazir, T.A., 2012. Learning to associate novel words with motor actions: language-induced motor activity following short training. Cortex 48 (7), 888-899.

Fischer, M.H., Zwaan, R.A., 2008. Embodied language: a review of the role of the motor system in language comprehension. Q. J. Exp. Psychol. 61 (6), 825-850.

Frith, C.D., Friston, K., Liddle, P.F., Frackowiak, R.S.J., 1991. Willed action and the prefrontal cortex in man: a study with PET. Proc. R. Soc. Lond. 244 (1311), 241-246.

Gastaut, H., 1952. Etude électrocorticographique de la réactivité des rythmes rolandiques. Rev. Neurol. 87, 176-182.

Glenberg, A.M., Gallese, V., 2011. Action-based language: a theory of language acquisition, comprehension, and production. Cortex 1, 1-18.

Godschalk, M., Lemon, R.N., Kuypers, H.G.J.M., Van der Steen, J., 1985. The involvement of monkey premotor cortex neurons in preparation of visually cued arm movements. Behav. Brain Res. 18 (2), 143-157.

Gross, J., Pollok, B., Dirks, M., Timmermann, L., Butz, M., Schnitzler, A., 2005. Task dependent oscillations during unimanual and bimanual movements in the human primary motor cortex and SMA studied with magnetoencephalography. Neuroimage 26 (1), 91-98.

Halsband, U., Freund, H.J., 1990. Premotor cortex and conditional motor learning in man. Brain 113 (1), 207-222.

Hauk, O., Pulvermüller, F., 2004. Neurophysiological distinction of action words in the fronto-central cortex. Hum. Brain Mapp. 21 (3), 191-201.

Jurkiewicz, M.T., Gaetz, W.C., Bostan, A.C., Cheyne, D., 2006. Post-movement beta rebound is generated in motor cortex: evidence from neuromagnetic recordings. Neuroimage 32 (3), 1281-1289.

Klimesch, W., Sauseng, P., Hanslmayr, S., Gruber, W., Freunberger, R., 2007. Event-related phase reorganization may explain evoked neural dynamics. Neurosci. Biobehav. Rev. 31 (7), 1003-1016.

Kuhlman, W.N., 1978. Functional topography of the human mu rhythm. Electroenceph. Clin. Neurophysiol. 44 (1), 83-93.

Kutas, M., Hillyard, S.A., 1984. Brain potentials during reading reflect word expectancy and semantic association. Nature 307 (5947), 161-163.

Liljestrom, M., Kujala, J., Jensen, O., Salmelin, R., 2005. Neuromagnetic localization of rhythmic activity in the human brain: a comparison of three methods. Neuroimage 25 (3), 734-745.

Mahon, B.Z., Caramazza, A., 2008. A critical look at the embodied cognition hypothesis and a new proposal for grounding conceptual content. J. Physiol. 102 (1), $59-70$.

Maris, E., Oostenveld, R., 2007. Nonparametric statistical of EEG- and MEG-data. J. Neurosci. Methods 164 (1), 177-190.

McFarland, D.J., Miner, L.A., Vaughan, T.M., Wolpaw, J.R., 2000a. Mu and Beta rhythm topographies during motor imagery and actual movements. Brain Topogr. 12 (3), 177-186.

McFarland, D.J., Miner, L.A., Vaughan, T.M., Wolpaw, J.R., 2000b. Mu and Beta Meaning. University Press, Oxford, UK

Mitra, P.P., Pesaran, B., 1999. Analysis of dinamic brain imaging data. Biophys. J. 76 (2), 691-708

Moody, C.L., Gennari, S.P., 2010. Effects of implied physical effort in sensory-motor and pre-frontal cortex during language comprehension. Neuroimage 49 (1), 782-793.

Moreno, I., de Vega, M., León, I., 2013. Understanding action language modulates oscillatory mu and beta rhythms in the same way as observing actions. Brain Cogn. 82 (3), 236-242.

Muthukumaraswamy, S.D., Johnson, B.W., McNair, N.A., 2004. Mu rhythm modulation during observation of an object-directed grasp. Cogn. Brain Res. 19 (2), 195-201. 
Nazir, T.A., Boulenger, V., Roy, A., Silver, B., Jeannerod, M., Paulignan, Y., 2008. Languageinduced motor perturbations during execution of a reaching movement. Q. J. Exp. Psychol. 61 (6), 933-943.

Neuper, C., Wörtz, M., Pfurtscheller, G., 2006. ERD/ERS patterns reflecting sensorimotor activation and deactivation. Prog. Brain Res. 159, 211-222.

Oberman, L.M., Hubbard, E.M., McCleery, J.P., Altschuler, E.L., Ramachandran, V.S., Pineda, J.A., 2005. EEG evidence for mirror neuron dysfunction in autism spectrum disorders. Cogn. Brain Res. 24 (2), 190-198.

Oostenveld, R., Praamstra, P., Stegeman, D.F., van Oosterom, A., 2001. Overlap of attention and movement-related activity in lateralized event-related brain potentials. Clin. Neurophysiol. 112 (3), 477-484.

Orgs, G., Dombrowski, J.H., Heil, M., Jansen-Osmann, P., 2008. Expertise in dance modulates alpha/beta event-related desynchronization during action observation. Eur. J. Neurosci. 27 (12), 3380-3384.

Papeo, L., Vallesi, A., Isaja, A., Rumiati, R.I., 2009. Effects of TMS on different stages of motor and non-motor verb processing in the primary motor cortex. PLoS ONE 4 (2), e4508.

Passingham, R.E., 1988. Premotor cortex and preparation for movement. Exp. Brain Res. 70 (3), 590-596.

Pfurtscheller, G., 1992. Event-related synchronization (ERS): an electrophysiological correlate of cortical areas at rest. Electroenceph. Clin. Neurophysiol. 83 (1), 62-69.

Pfurtscheller, G., Lopes da Silva, F.H., 1999. Event-related EEG/MEG synchronization and desynchronization: basic principles. Clin. Neurophysiol. 110, 1842-1857.

Pfurtscheller, G., Neuper, C., Andrew, C., Edlinger, G., 1997. Foot and hand area mu rhythms. Int. J. Psychophysiol. 26 (1-3), 121-135.

Pfurtscheller, G., Brunner, C., Schlögl, A., Lopes de Silva, F.H., 2006. Mu rhythm (de) synchronization and EEG single-trial classification of different motor imagery tasks. Neuroimage 31 (1), 153-159.

Pineda, J.A., 2005. The functional significance of mu rhythms: translating "seeing" and "hearing" into "doing". Brain Res. Rev. 50 (1), 57-68.

Polich, J., 2007. Updating P300: an integrative theory of P3a and P3b. Clin. Neurophysiol. 118 (10), 2128-2148.

Pulvermüller, F., 1996. Hebb's concept of cell assemblies and the psychophysiology of word processing. Psychophysiol. 33, 317-333.

Pulvermüller, F., Lutzenberger, W., Preissl, H., 1999. Nouns and verbs in the intact brain: evidence from event-related potentials and high-frequency cortical responses. Cereb. Cortex 9 (5), 497-506

Pulvermüller, F., Shtyrov, Y., Ilmoniemi, R.J., 2005. Brain signatures of meaning access in action word recognition. J. Cogn. Neurosci. 17, 884-892.

Pulvermüller, F., Shtyrov, Y., Hauk, O., 2009. Understanding in an instant. Neurophysiological evidence for mechanistic language circuits in the brain. Brain Lang. 110 (2), 81-94.

Quin, L., He, B., 2005. A wavelet-based time-frequency analysis approach for classification of motor imagery for brain-computer interface applications. J. Neural Eng. 2, 65-72.

Raposo, A., Moss, H.E., Stamatakis, E.A., Tyler, L.K., 2009. Modulation of motor and premotor cortices, by actions, actions words and action sentences. Neurophysiologia 47 (2), 388-396.

Reinvang, R., 1999. Cognitive event-related potentials in neuropsychological assessment. Neuropsychol. Rev. 9 (4), 231-248.

Rizzolatti, G., Arbib, M.A., 1998. Language within our grasp. Trends Neurosci. 21 (5), 188-194.
Rizzolatti, G., Craighero, L., 2004. The mirror-neuron system. Annu. Rev. Neurosci. 27 169-192.

Rizzolatti, G., Luppino, G., 2001. The cortical motor system. Neuron 31 (6), 889-901.

Rueschemeyer, S., Rooij, D., Lindemann, O., Willems, R.M., Bekkering, H., 2010. The function of words: distinct neural correlates for words denoting differently manipulable objects. J. Cogn. Neurosci. 22 (8), 1844-1851.

Sato, M., Mengarelli, M., Riggio, L., Gallese, V., Buccino, H., 2008. Task related modulation of the motor system during language processing. Brain Lang. 105 (2), 83-90.

Schneider, W., Eschmann, A., Zuccolotto, A., 2002. E-Prime v1.1. Psychology Software Tools Inc., Pittsburgh, PA.

Schuil, K.D.I., Smits, M., Zwaa, R.A., 2013. Sentential context modulates the involvement of the motor cortex in action language processing: an fMRI study. Front. Hum. Neurosci. 7, 100.

Smith, J.L., Johnstone, S.J., Barrv, R.J., 2008. Movement related potentials in Go/No Go task: the P3 reflects both cognitive and motor inhibition. Clin. Neurophysiol. 119, 704-714.

Taylor, L.J., Lev-Ari, S., Zwaan, R., 2008. Inferences about action engage systems. Brain Lang. 107 (1), 62-67.

Tettamanti, M., Buccino, G., Saccuman, M.C., Gallese, V., Danna, M., Scifo, P., 2005 Listening to action-related sentences activates fronto-parietal motor circuits. J. Cogn. Neurosci. 17 (2), 273-281.

Tomasino, B., Fink, G.R., Sparing, R., Dafotakis, M., Weiss, P.H., 2008. Action verbs and the primary motor cortex: a comparative TMS study of silent reading, frequency judgments, and motor imagery. Neuropsychologia 46 (7), 1915-1926.

Urgesi, C., Calvo-Merino, B., Haggard, P., Aglioti, S.M., 2007. Transcranial magnetic stimulation reveals two cortical pathways for visual body processing. J. Neurosci. 27 (30), 8023-8030.

Urrutia, M., Gennari, S.P., de Vega, M., 2012. Counterfactuals in action: an fMRI study of counterfactual sentences describing physical effort. Neuropsychologia 50 (14) 3663-3672.

van Elk, M., van Schie, H.T., Zwaan, R.A., Bekkering, H., 2010. The functional role of motor activation in language processing: motor cortical oscillations support lexical-semantic retrieval. Neuroimage 50 (2), 665-677.

Wang, L., Jensen, O., Brink, D., Weder, N., Schoffelen, J.-M., Magyari, L., Hagoort, P. Bastiaansen, M., 2012. Beta oscillations relate to the N400m during language comprehension. Hum. Brain Mapp. 33 (12), 2898-2912.

Weiner, K.S., Grill-Spector, K., 2011. Not one extrastriate body area: using anatomica landmarks, Hmt +, and visual field maps to parcellate limb-selective activations in human lateral occipitotemporal cortex. Neuroimage 56 (4), 2183-2199.

Weiss, S., Mueller, H.M., 2012. "Too many betas do not spoil the broth": the role of beta brain oscillations in language processing. Front. Psychol. 3, 201.

Weisz, N., Hartmann, T., Müller, N., Lorenz, I., Obleser, J., 2011. Alpha rhythms in audition: cognitive and clinical perspectives. Percept. Sci. 2, 73.

Willems, R.M., Hagoort, P., Casasanto, D., 2010. Body-specific representations of action verbs: neural evidence from right- and left-handers. Psychol. Sci. 21, 67-74.

Woertz, M., Pfurtscheller, G., Klimesch, W., 2004. Alpha power dependent light stimulation: dynamics of event-related (de) synchronization in human electroencephalogram. Cogn. Brain Res. 20 (2), 256-260. 